International Journal of Engineering \& Technology, $7(3.31)(2018)$ 179-185
International Journal of Engineering \& Technology
SPC
Website: www.sciencepubco.com/index.php/IJET
Research paper

\title{
Asymptotic Stability of Solution of Lyapunov Type Matrix Volterra Integro-Dynamic System on Time Scales
}

\author{
G.V. Ramana ${ }^{1}$, G.V. S. R. Deekshitulu ${ }^{2} *$ \\ ${ }^{1}$ Department of Mathematics, \\ Aditya Engineering College, Surampalem, A.P., India. \\ ${ }^{2}$ Department of Mathematics, \\ UCEK, JNTUK, Kakinada, A.P., India. \\ *Corresponding author E-mail: dixitgvsr@hotmail.com
}

\begin{abstract}
This article emphasizes the characteristics and nature of asymptotic stability of solution of Lyapunov type matrix Volterra integrodynamic system on time scales.
\end{abstract}

Keywords: Asymptotic stability; Lyapunov; integro systems; time scales.

\section{Introduction}

Integro-differential equations occur as mathematical models in mechanics, mathematical biology and many other diverse disciplines quite frequently. The origin of the study of integral and integro differential equations may be traced back to the works of Lotka, Fredholm, Volterra etc [9]. From these initial steps, the theory and applications of integro-differential equations have emerged as new area of investigation. Later the study of qualitative properties of Integro-differential equation has drawn the attention of many mathematicians. Burton studied the stability theory of Volterra integro-differential equations [2, 3]. Grossman and Miller observed the asymptotic behaviour of solutions of Volterra integro-differential system [4].

The theory, proposed by Hilger [5], of time scales as a tool to unify the discrete and continuous calculus, is now a well established subject. For further study on dynamic equations, inequalities, linear system of equations on time scales, one can refer [1] and reference there in. In [7], basic properties of quantitave and qualitative results for Volterra integral equations on time scales were introduced by Kulik and Tindell.. Recently, Lupulescu, Ntouyas and Younus have discussed the asymptotic stability and boundedness of Volterra integro-differential equations on time scales [8]. The importance of lyapunov type system is useful in many branches of Science and Technology and particularly in Control theory and Systems Engineering. Inspired by the quite interesting nature of this problem, an effort, to study the asymptotic stability for the system given below, is made.

$$
\begin{aligned}
\mathrm{X}^{a_{(}}(t)= & A(t) \mathrm{X}(t)+\mathrm{X}(t) B(t) \\
& +\int_{t_{0}}^{t}\left[\mathrm{~K}_{1}(t, s) \mathrm{X}(s)+\mathrm{X}(s) \mathrm{K}_{2}\left(t_{s} s\right)\right] \Delta s+F(t), \\
\mathrm{X}\left(t_{0}\right)= & \mathrm{X}_{0^{*}}
\end{aligned}
$$

where $0 \leq t_{0} \in \mathbb{T}^{k}$ is fixed, $A, B$ and $F$ are an $(n \times n)$ continuous matrix functions on $\mathbb{T}, \mathbb{K}_{1}(t, s)$ and $\mathbb{K}_{2}(t, s)$ are an $(n \times n)$

continuous matrix functions on

$\Omega=\left\{\left(t_{,} s\right) \in \mathbb{T} \times \operatorname{Ti} t_{0} \leq s \leq t<\infty\right\}$

\section{Preliminary Results}

Throught this paper $\mathbb{T}$ denotes time scale( closed arbitray and nonempty subset of the real numbers).

Definition 2.1: ([1]) The mappings $\sigma$ and $p \times \mathbb{T} \rightarrow \mathbb{R}$ where $\mathbb{T}$ is any closed subset of reals, are defined as $\sigma(t)=\inf \{s \in \mathbb{T}: s>t\}$ and $p(t)=\sup \{s \in \mathbb{T}: s<t\}$.

Definition 2.2: ([1]) A non-maximal element $t$ in $\mathbb{T}$ is called right dense if $\sigma(t)=t$; right scattered if $\sigma(t)>t$; left dense if $\rho(t)=t$ and left scattered if $\rho(t)<t$.

Definition 2.3: ([1]) If $\mathbb{T}$ has a left scattered maximum $M$, then $\mathbb{T}^{K}=\mathbb{T}-\{M\}$, otherwise, $\mathbb{T}^{K}=\mathbb{T}$. If $\mathbb{T}$ has a right scattered minimum $m$, then $\mathbb{T}^{k}=\mathbb{T}-\{m\}$, otherwise, $\mathbb{T}^{k}=\mathbb{T}$.

Definition 2.4: ([1]) The function $\mu^{*}: \mathbb{T}^{K} \rightarrow \mathbb{R}^{+}$defined by $\mu^{*}(t)=\mu(\sigma(t), t)$ for $t \in \mathbb{T}$ is said to be graininess. If $t$ is right dense, then $\mu^{3}=0$ and if $t$ is right scattered, then $\mu^{*}=\sigma(t)-t$.

Definition 2.5: ([1]) A functions $f: \mathbb{T} \rightarrow \mathbb{R}$ is said to be differentiable at $t \in \mathbb{T}$, if there exists an $\alpha \in \mathbb{R}$ such that for any $\varepsilon>0$ there exists a neighbourhood $N$ of $t$ satisfying $|f(\sigma(t))-f(s)-(\sigma(t)-s) \alpha| \leq|\sigma(t)-s|$ for all $s \in N$.

Theorem 2.6: ([1]) If $A$ is differentiable at $t \in \mathbb{T}^{K}{ }_{x}$ then $A^{\sigma}(t)=A(t)+\mu(t) A^{A}(t)$.

(1) Theorem 2.7: ([1]) Suppose $A, B$ and $C$ are differentiable $(n \times n)$ 
matrix-valued functions. Then

(a) $(A+B)^{\Delta}=A^{\Delta}+B^{A}$

(b) $(\alpha A)^{\Delta}=\alpha A^{\Delta}$ if $\alpha$ is constant;

(c) $(A B)^{\Delta}=A^{\unlhd} B^{\sigma}+A B^{\Delta}=A^{\sigma} B^{\Delta}+A^{\unlhd} B_{i}$

(d) $(A B C)^{\Delta}=A^{\lrcorner} B C+A^{\sigma} B^{\Delta} C^{\sigma}+A^{\sigma} B C^{\Delta}=A^{\Delta} B C+A^{\sigma} B^{\Delta} C$ $+A^{\sigma} B^{\sigma} C^{\Theta}$.

Lemma 2.8: ([1]) If $p, q \in C_{r d}\left(\mathbb{T}_{v}, \mathbb{R}\right)$, Then

$$
e_{p ! q}^{\Delta}\left(x, t_{0}\right)=(p-q) \frac{e_{p}\left(x, t_{0}\right)}{e_{q}^{\sigma}\left(x, t_{0}\right)}
$$

Lemma 2.9: ([1]) Let $\alpha \in \mathbb{R}$ with $\alpha \in \mathbb{R}^{+}$. Then $e_{\alpha}(t, s) \geq 1+\alpha(t-s)$ for all $t \geq s$.

Lemma 2.10: ([1]) Let $y \in C_{r d}\left(\mathbb{T}_{v}, \mathbb{R}\right), p \in \mathbb{R}^{+}, p \geq 0$ and $\alpha \in \mathbb{R}$. Then

$y(t) \leq \alpha+\int_{t_{0}}^{\pi} y(s) p(s) \Delta s$

for all $t \in \mathbb{T}_{x}$ implies

for all $t \in \mathbb{T}_{x}$

$y(t) \leq \alpha e_{p}\left(t, t_{0}\right)$

Theorem 2.11: ([6], Fubini's theorem) Let $a, b \in \mathbb{T}$ with $b>a$ and it is assumed that $f: \mathbb{T} \times \mathbb{T} \rightarrow \mathbb{R}$ is integrable on $\left\{(t, s) \in \mathbb{T} \times T_{i} b>t>s \geq a\right\}$. Then

$$
\int_{\mathbb{E}}^{\infty} \int_{\mathbb{E}}^{\infty} f(s, u) \Delta u \Delta s=\int_{\mathbb{E}}^{\infty} \int_{\sigma(s)}^{D} f(s, u) \Delta s \Delta u
$$

\section{Main Results}

Theorem 3.1: If $\mathrm{L}_{1}(t, s)$ and $\mathrm{L}_{2}(t, s)$ are continuously differentiable $(n \times n)$ matrix functions on $\Omega$ such that (i) they commute with $\bar{X}(t)$ and (ii) they satisfies

$$
\begin{aligned}
& \mathbb{K}_{1}(t, s)+\mathbb{L}_{i s}^{s}(t, s)+\mathbb{L}_{1}\left(t_{3} \sigma(s)\right) A(s)+A(s) \mathbb{L}_{2}(t, \sigma(s)) \\
& +\int_{\sigma}^{\mathbb{R}}\left[\mathrm{L}_{1}\left(\mathrm{t}_{2} \sigma(\tau)\right) \mathbb{K}_{1}\left(\tau_{2} s\right)+\mathbb{K}_{1}\left(\tau_{2} s\right) \mathrm{L}_{2}\left(t_{2} \sigma(\tau)\right)\right] \Delta \tau=0 \\
& \mathbb{K}_{2}\left(t_{2} s\right)+\mathbb{L}_{2}^{\beta}\left(t_{2} s\right)+\mathrm{L}_{1}\left(t_{2} \sigma(s)\right) B(s)+B(s) \mathrm{L}_{2}\left(t_{2} \sigma(s)\right)
\end{aligned}
$$

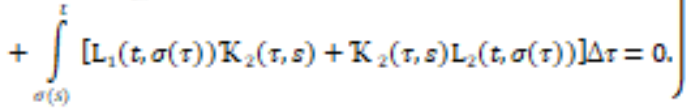

Then the equation (1) is equivalent to the following system

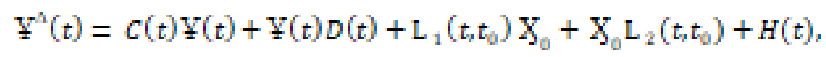

$\mathrm{Y}\left(\mathrm{t}_{0}\right)=\mathrm{X}_{0^{x}}$

$\underset{C(t)}{\text { where }}=A(t)-\mathrm{L}_{1}(t, t)$,

$D(t)=B(t)-L_{2}(t, t)$,

$\left.H(t)=F(t)+\int_{t_{0}}^{t}\left[\mathrm{~L}_{1}(t, \sigma(s)) F(s)+F(s) \mathrm{L}_{2}(t, \sigma(s))\right] \Delta s_{v}\right\}$

where $C(t), D(t)$ and $H(t)$ are $(n \times n)$ continuous matrix functions on $\mathbb{T}$.

\section{Proof:}

Let $X(t)$ be any solution of (1) on $\mathbb{T}$.

$$
\text { Set } P(s)=L_{1}(t, s) \mathrm{X}(s)+\mathrm{X}(s) L_{2}(t, s)
$$

$$
\begin{aligned}
& P^{\Delta}(s)=L_{1 s}^{\Delta}\left(t_{s} s\right) \mathrm{X}(s)+L_{1}\left(t_{s} \sigma(s)\right) \mathrm{X}^{\Delta}(s)+\mathrm{X}(s) L_{2 s}^{\Delta}\left(t_{s} s\right) \\
& +\mathrm{X}^{\mathrm{A}}(s) L_{2}(\mathrm{t}, \sigma(s)) \text {. } \\
& \left.+\left[\int_{0}^{s} \mathbb{K}_{1}(s, \tau) \mathrm{X}(\tau)\right) \Delta \tau\right] \mathrm{L}_{2}\left(t_{2} \sigma(s)\right)+\mathbb{L}_{1}\left(t_{2} \sigma(s)\right) F(s) \\
& +\mathrm{X}(s) \mathrm{L}_{2}^{\mathrm{s}}\left(\mathrm{t}_{s} s\right)+\mathrm{X}(s) B(s) \mathrm{L}_{i}\left(\mathrm{t}_{s} \sigma(s)\right)+\mathrm{X}(s) \mathrm{L}_{1}\left(\mathrm{t}_{s} \sigma(s)\right) B(s) \\
& \left.\left.+\mathrm{L}_{1}\left(t_{2} \sigma(s)\right)\left[\int_{\omega_{w}}^{s} \mathrm{X}(\tau)\right) \mathrm{K}_{2}(s, \tau)\right) \Delta \mathrm{r}\right] \\
& \left.\left.+\left[\int_{\omega_{1}}^{s} \mathrm{X}(\tau)\right) \mathbb{K}_{2}(s, \tau)\right) \Delta \mathrm{r}\right] \mathrm{L}_{-}(\mathrm{t}, \sigma(s))+F(s) \mathrm{L}_{2}\left(\mathrm{t}_{3} \sigma(s)\right)
\end{aligned}
$$

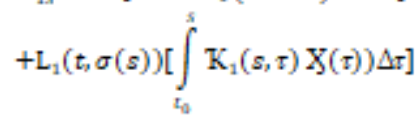

Integrating between $t_{0}$ to $t$ and using Fubini's Theorem, we have

$$
\begin{aligned}
& P(\mathrm{t})-P\left(\mathrm{t}_{\mathrm{a}}\right)=\int_{\omega_{\mathrm{W}}}^{\mathrm{T}}\left[\mathrm{L}_{\mathrm{H}}^{\mathrm{Q}}\left(\mathrm{t}_{2} s\right)+\mathrm{L}_{1}\left(\mathrm{t}_{2} \sigma(s)\right) A(s)\right. \\
& +A(s) \mathrm{L}_{2}\left(t_{2} \sigma(s)\right)+\int_{\omega|s\rangle}^{\mathrm{T}} \mathrm{L}_{1}\left(\mathrm{t}_{2} \sigma(u)\right) \mathbb{K}_{1}(u, s) \Delta u \\
& \left.+\int_{\omega[s p}^{\pi} \mathbb{K}_{1}(u, s) \mathrm{L}_{2}(\mathrm{t}, \sigma(u)) \Delta u\right] \mathrm{X}(s) \Delta s \\
& +\int_{\mathbb{L}_{1}}^{\mathrm{r}} \mathrm{L}_{1}(\mathrm{t}, \sigma(s)) F(s) \Delta s+\int_{\mathbb{L}_{2}}^{\mathrm{r}} F(s) \mathrm{L}_{2}\left(\mathrm{t}_{2} \sigma(s)\right) \Delta s \\
& +\int_{\omega_{10}}^{\mathrm{F}} \mathrm{X}(s)\left[\mathrm{L}_{2}^{\mathrm{a}}(\mathrm{t}, s)+\mathrm{L}_{1}(\mathrm{t}, \sigma(s)) B(s)\right.
\end{aligned}
$$

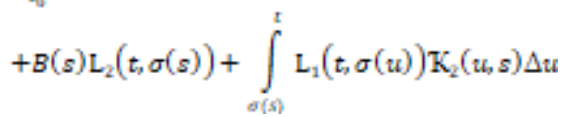

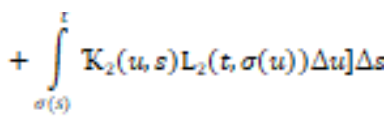

From (2), we have

$$
\begin{aligned}
& L_{1}\left(t_{2} t\right) X(t)+X(t) L_{2}\left(t_{2} t\right) \\
& =L_{1}\left(t_{3} t_{0}\right) \mathrm{X}\left(\mathrm{t}_{2}\right)+\mathrm{X}\left(\mathrm{t}_{0}\right) L_{2}\left(\mathrm{t}_{2} \mathrm{t}_{2}\right) \\
& +\int_{t_{0}}^{\pi}\left[L_{1}\left(t_{s} \sigma(s)\right) F(s)+F(s) L_{2}\left(t_{j} \sigma(s)\right)\right] \Delta s \\
& -\int_{t_{0}}^{t} \mathbb{K}_{1}(t, s) \mathrm{X}(s) \Delta s-\int_{t_{0}}^{t} \mathrm{X}(s) \mathrm{K}_{2}(t, s) \Delta s
\end{aligned}
$$

From (1) and (4), we have

$X^{n}(t)=c(t) X(t)+X(t) D(t)+L_{1}\left(t_{2} t_{0}\right) X_{0}+X_{0} L_{2}\left(t_{2} t_{0}\right)+H(t)$

Hence $\mathrm{X}(t)$ satisfies (3).

Conversely, suppose that $Y(t)$ is any solution of (3) on $\mathbb{T}$ and commute with $L_{1}(t, s)$ and $L_{2}(t, s)$.

Consider

$$
\begin{aligned}
& Z(t)=Y^{\Delta}(t)-F(t)-A(t) \Psi(t)-Y(t) B(t) \\
& -\int_{t_{0}}^{t}\left[\mathbb{K}_{1}(t, s) \mathrm{Y}(s)+\mathrm{Y}(s) \mathbb{K}_{2}(t, s)\right] \Delta s
\end{aligned}
$$


From (2), (3), (4) and using Fubini's Theorem, we have

$$
\begin{aligned}
& z(t)=-\int\left[L_{1}(t, t) Y(t)+Y(t) L_{2}(t, t)\right] \\
& \left.-\left[L_{1}\left(t_{1} t_{0}\right) \mathrm{X}\left(\mathrm{t}_{0}\right)+\mathrm{X}\left(\mathrm{t}_{2}\right) L_{2}\left(\mathrm{t}_{2} \mathrm{t}_{0}\right)\right]\right\} \\
& +\int_{\sigma_{1}}^{\mathrm{F}}\left[L_{1}\left(\mathrm{t}_{1} \sigma(s)\right) F(s)+F(s) L_{2}\left(\mathrm{t}_{2} \sigma(s)\right)\right] \Delta s
\end{aligned}
$$

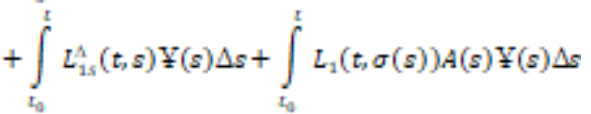

$$
\begin{aligned}
& +\int_{\mathbb{E}_{2}}^{\mathrm{E}} A(s) L_{2}\left(\mathrm{t}_{2} \sigma(s)\right) \mathrm{Y}(\mathrm{s}) \\
& +\int_{\mathbb{L}_{1}}^{\mathrm{T}} L_{1}\left(t_{2} \sigma(s)\right)\left[\int_{\mathbb{L}_{1}}^{\infty} \mathbb{K}_{1}(s, u) Y(u) \Delta u\right] d s
\end{aligned}
$$

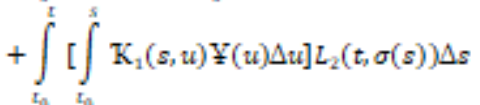

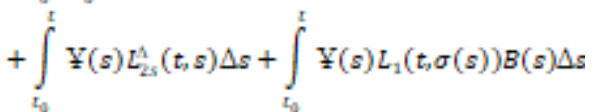

$$
\begin{aligned}
& +\int^{\pi} Y(s) B(s) L_{i}(t, \sigma(s)) d s \\
& +\int_{\mathbb{L}_{1}}^{\mathbb{R}} L_{1}\left(t_{2} \sigma(s)\right)\left[\int_{\mathbb{L}_{1}}^{\infty} Y(u) \mathbb{K}_{2}(s, u) \Delta u\right] d s
\end{aligned}
$$

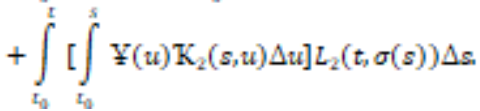

$$
\begin{aligned}
\text { Set } Q(s)= & L_{1}(t, s) Y(s)+Y(s) L_{2}(t, s) \\
Q^{\Delta}(s)= & L_{1 s}^{\Delta}(t, s) Y(s)+L_{1}(t, \sigma(s)) \Psi^{\Delta}(s) \\
& +Y(s) L_{2 s}^{\Delta}(t, s)+Y^{\Delta}(s) L_{2}(t, \sigma(s))
\end{aligned}
$$

Integrating between $t_{0}$ to $t$, we have

$$
\begin{aligned}
& Q(t)-Q\left(t_{0}\right)=\int_{t_{0}}^{\pi}\left[L_{1 s}^{\Delta}\left(t_{s} s\right) \mathrm{Y}(s)+L_{1}(t, \sigma(s)) \Psi^{\Delta}(s)\right. \\
& \left.+\mathrm{X}(s) L_{2 s}^{\Delta}(t, s)+\mathrm{Y}^{\Delta}(s) L_{2}(t, \sigma(s))\right] \Delta s \\
& {\left[L_{1}(t, t) \mathrm{Y}(t)+\mathrm{Y}(t) L_{2}(t, t)\right]-\left[L_{1}\left(t, t_{0}\right) \mathrm{X}_{0}+\mathrm{X}_{0} L_{2}\left(t, t_{0}\right)\right]} \\
& =\int_{t_{0}}^{t}\left[L_{1 s}^{\Delta}(t, s) Y(s)+L_{1}(t, \sigma(s)) \Psi^{\Delta^{a}}(s)\right. \\
& \left.+\mathrm{Y}(s) L_{2 s}^{\Delta}(t, s)+\mathrm{Y}^{\Delta}(s) L_{2}(t, \sigma(s))\right] \Delta s
\end{aligned}
$$

(6) Theorem 3.2: If $L_{1}(t, s)$ and $L_{2}(t, s)$ are continuously differentiable $(n \times n)$ matrix functions on $\Omega$ and commute with $X(t)$. Then the equation (1) is equivalent to the following system

$$
\begin{aligned}
& Y^{3}(t)=c(t) Y(t)+Y(t) D(t)+\int_{L_{12}}^{L}\left[G_{1}\left(t_{2} s\right) Y(s)+Y(s) G_{2}(t, s)\right] \Delta s+H(t) \\
& Y\left(t_{0}\right)=X_{Q^{a}}
\end{aligned}
$$

Where

$$
\left.\begin{array}{rl}
C(t)= & A(t)-L_{1}(t, t), \\
D(t)= & B(t)-L_{2}(t, t), \\
H(t)= & F(t)+L_{1}\left(t, t_{0}\right) \mathrm{X}_{0}+\mathrm{X}_{0} L_{2}\left(t, t_{0}\right) \\
& +\int_{t_{0}}^{t}\left[L_{1}(t, \sigma(s)) F(s)+F(s) L_{2}(t, \sigma(s))\right] \Delta s
\end{array}\right\}
$$

and

On substituting (7) in (6), we have

$$
\begin{aligned}
& \begin{aligned}
Z(t)= & -\int_{t_{0}}^{t} L_{1}\left(t_{v} \sigma(s)\right) X \Psi^{\Delta}(s)-F(s)-A(s) \mathrm{Y}(s)- \\
& \left.-\int_{t_{0}}^{s}\left[\mathrm{~K}_{1}(s, u) \mathrm{Y}(u)+\mathrm{Y}(u) \mathbb{K}_{2}(s, u)\right] \Delta u\right) \Delta s
\end{aligned} \\
& -\int_{t_{0}}^{t}(Y(s)-F(s)-A(s) Y(s)-Y(s) B(s) \\
& \left.-\int_{t_{0}}^{s}\left[\mathrm{~K}_{1}(s, u) \Psi(u)+\mathbb{Y}(u) \mathbb{K}_{2}(s, u)\right] \Delta u\right\} L_{2}(t, \sigma(s)) \Delta s
\end{aligned}
$$

From (5), we have
$\left.\begin{array}{rl}G_{1}(t, s)= & \mathbb{K}_{1}(t, s)+L_{1 s}^{\Delta}(t, s)+L_{1}(t, \sigma(s)) A(s)+A(s) L_{2}(t, \sigma(s)) \\ & +\int_{\sigma[(s)}^{t}\left[L_{1}(t, \sigma(\tau)) \mathbb{K}_{1}\left(\tau_{s} s\right)+\mathbb{K}_{1}(\tau, s) L_{2}(t, \sigma(\tau))\right] \Delta \tau_{v} \\ G_{2}(t, s) B(s) & =\mathbb{K}_{2}(t, s)+L_{2 s}^{\Delta}(t, s)+L_{1}(t, \sigma(s)) B(s)+B(s) L_{2}(t, \sigma(s)) \\ & +\int_{\sigma(s)}^{t}\left[L_{1}(t, \sigma(\tau)) \mathbb{K}_{2}\left(\tau_{s} s\right)+\mathbb{K}_{2}(\tau, s) L_{2}(t, \sigma(\tau))\right] \Delta \tau_{x}\end{array}\right\}$

Where $C(t), D(t)$ and $H(t)$ are $(n \times n)$ continuous matrix functions on $\mathbb{T}$ and $G_{1}(t, s), G_{2}(t, s)$ are $(n \times n)$ matrix functions on $\Omega$ and commute with $\Psi(t)$.

\section{Proof:}

Let $\mathrm{X}(\mathrm{t})$ be any solution of (1) on $\mathbb{T}$.

Set $P(s)=L_{1}(t, s) \mathrm{X}(s)+\mathrm{X}(s) L_{2}(t, s)$ 


$$
\begin{aligned}
P^{\Delta}(s)= & L_{1 s}^{\Delta}\left(t_{s} s\right) \mathrm{X}(s)+L_{1}(t, \sigma(s)) \mathrm{X}^{\lrcorner}(s) \\
& +\mathrm{X}(s) L_{2 s}^{\Delta}\left(t_{s} s\right)+\mathrm{X}^{\Delta}(s) L_{2}(t, \sigma(s)) .
\end{aligned}
$$

From (1), we have

$$
\begin{aligned}
P^{\Delta}(s)= & L_{1 s}^{\Delta}(t, s) \mathrm{X}(s)+L_{1}(t, \sigma(s)) A(s) \mathrm{X}(s) \\
& +A(s) L_{2}(t, \sigma(s)) \mathrm{X}(s) \\
& \left.+L_{1}(t, \sigma(s))\left[\int_{t_{0}} \mathbb{K}_{1}(s, \tau) \mathrm{X}(\tau)\right) \Delta \tau\right] \\
& \left.+\left[\int_{t_{0}}^{s} \mathrm{~K}_{1}(s, \tau) \mathrm{X}(\tau)\right) \Delta \tau\right] L_{2}(t, \sigma(s)) \\
& +L_{1}(t, \sigma(s)) F(s) \\
& +\mathrm{X}(s) L_{2 s}^{\Delta}(t, s)+\mathrm{X}(s) B(s) L_{2}(t, \sigma(s)) \\
& +\mathrm{X}(s) L_{1}(t, \sigma(s)) B(s) \\
& \left.\left.+L_{1}(t, \sigma(s))\left[\int_{t_{0}} \mathrm{X}(\tau)\right) \mathbb{K}_{2}(s, \tau)\right) \Delta \tau\right] \\
& \left.\left.\left.+\left[\int_{t_{0}} \mathrm{X}(\tau)\right)\right) \mathbb{K}_{2}(s, \tau)\right) \Delta \tau\right] L_{2}(t, \sigma(s)) \\
& +F(s) L_{2}(t, \sigma(s))
\end{aligned}
$$

Integrating between $t_{0}$ to $t$ and using Fubini's Theorem, we have

$$
\begin{aligned}
& P(t)-P\left(t_{0}\right)=\int_{t_{0}}^{\pi}\left[L_{1 s}^{\Delta}\left(t_{s} s\right)+L_{1}(t, \sigma(s)) A(s)+A(s) L_{2}(t, \sigma(s))\right. \\
& +\int_{\sigma[s)}^{t} L_{1}(t, \sigma(u)) \mathbb{K}_{1}(u, s) \Delta u \\
& \left.+\int_{\sigma[s)}^{t} \mathbb{K}_{1}(u, s) L_{2}(t, \sigma(u)) \Delta u\right] \mathrm{X}(s) \Delta s \\
& +\int_{t_{0}}^{t} L_{1}(t, \sigma(s)) F(s) \Delta s+\int_{t_{0}}^{t} F(s) L_{2}(t, \sigma(s)) \Delta s \\
& +\int_{t_{0}}^{t} \mathrm{X}(s)\left[L_{2 s}^{\Delta}\left(t_{s} s\right)+L_{1}(t, \sigma(s)) B(s)\right. \\
& +B(s) L_{2}(t, \sigma(s))+\int_{\sigma[s)}^{t} L_{1}(t, \sigma(u)) \mathrm{K}_{2}(u, s) \Delta u \\
& \left.+\int_{\sigma(s)}^{t} K_{2}(u, s) L_{2}(t, \sigma(u)) \Delta u\right] \Delta s \\
& P(t)-P\left(t_{0}\right)=\int_{t_{0}}^{\pi}\left[L_{1 s}^{\Delta}\left(t_{s} s\right)+L_{1}(t, \sigma(s)) A(s)+A(s) L_{2}(t, \sigma(s))\right. \\
& +\int_{\sigma(s)}^{t} L_{1}(t, \sigma(u)) \mathbb{K}_{1}(u, s) \Delta u \\
& \left.+\int_{\sigma[s)}^{t} \mathbb{K}_{1}(u, s) L_{2}(t, \sigma(u)) \Delta u\right] \mathrm{X}(s) \Delta s \\
& +\int_{t_{0}}^{t} L_{1}(t, \sigma(s)) F(s) \Delta s+\int_{t_{0}}^{t} F(s) L_{2}(t, \sigma(s)) \Delta s \\
& +\int_{t_{0}}^{t} \mathrm{X}(s)\left[L_{2 s}^{\Delta}(t, s)+L_{1}(t, \sigma(s)) B(s)\right.
\end{aligned}
$$

$$
\begin{aligned}
& +B(s) L_{2}(t, \sigma(s))+\int_{\sigma(s)}^{\pi} L_{1}\left(t_{s} \sigma(u)\right) K_{2}(u, s) \Delta u \\
& \left.+\int_{\sigma[s)}^{t} \mathbb{K}_{2}(u, s) L_{2}(t, \sigma(u)) \Delta u\right] \Delta s
\end{aligned}
$$

From (10), we have

$L_{1}(t, t) \mathrm{X}(t)+\mathrm{X}(t) L_{2}(t, t)$

$$
\begin{aligned}
& =L_{1}\left(t_{s} t_{0}\right) \mathrm{X}\left(t_{0}\right)+\mathrm{X}\left(t_{0}\right) L_{2}\left(t_{s} t_{0}\right) \\
& \quad+\int_{t_{0}}^{t}\left[L_{1}(t, \sigma(s)) F(s)+F(s) L_{2}(t, \sigma(s))\right] \Delta s \\
& \quad+\int_{t_{0}}^{t} G_{1}(t, s) \mathrm{X}(s) \Delta s-\int_{t_{0}}^{t} \mathrm{~K}_{1}(t, s) \mathrm{X}(s) \Delta s \\
& \quad+\int_{t_{0}}^{t} \mathrm{X}(s) G_{2}(t, s) \Delta s-\int_{t_{0}}^{t} \mathrm{X}(s) \mathrm{K}_{2}(t, s) \Delta s
\end{aligned}
$$

From (9), we have

$$
\begin{aligned}
& {[A(t)-C(t)] \mathrm{X}(t)+\mathrm{X}(t)[B(t)-D(t)]} \\
& =H(t)-F(t)+\int_{t_{0}}^{t}\left[G_{1}(t, s) \mathrm{X}(s)+\mathrm{X}(s) G_{2}(t, s)\right] \Delta s \\
& -\int_{t_{0}}^{t}\left[\mathrm{~K}_{1}(t, s) \mathrm{X}(s)+\mathrm{X}(s) \mathrm{K}_{2}\left(t_{s} s\right)\right] \Delta s \\
& A(t) \mathrm{X}(\mathrm{t})+\mathrm{X}(\mathrm{t}) B(\mathrm{t})+\int_{\mathbb{w}_{1}}^{\mathrm{T}}\left[\mathrm{K}_{1}\left(t_{t} s\right) \mathrm{X}(s)+\mathrm{X}(s) \mathrm{K}_{\mathcal{L}}(t, s)\right] \Delta s+F(t) \\
& =c(t) \mathrm{X}(\mathrm{t})+\mathrm{X}(\mathrm{t}) D(\mathrm{t})+\int_{\omega_{1}}^{\mathrm{T}}\left[G_{1}\left(t_{2} s\right) \mathrm{X}(s)+\mathrm{X}(s) G_{2}\left(t_{2} s\right)\right] \Delta s \\
& +H(t)
\end{aligned}
$$

From (1), we have

$\mathrm{X}^{\mathrm{s}}(\mathrm{t})=C(\mathrm{t}) \mathrm{X}(\mathrm{t})+\mathrm{X}(\mathrm{t}) D(\mathrm{t})+\int_{\tau_{1}}^{\mathrm{L}}\left[G_{1}\left(t_{t} s\right) \mathrm{X}(s)+\mathrm{X}(s) G_{2}\left(t_{2} s\right)\right] \Delta s+H(\mathrm{t})$ Hence $\mathrm{X}(t)$ satisfies (8).

Conversely, suppose that $Y(t)$ is any solution of (8) on $\mathbb{T}$ and commute with $L_{1}(t, s)$ and $L_{2}(t, s)$.

Consider

$z(t)=\Psi^{2}(t)-F(t)-A(t) \Psi(t)-Y(t) B(t)-\int_{\omega_{1}}^{t}\left[\mathbb{K}_{1}\left(t_{2} s\right) \Psi(s)+Y(s) \mathbb{K}_{2}\left(t_{2} s\right)\right] \Delta s$

From (8), (9), (10) and using Fubini's Theorem, we have

$z(t)=-\left\{\left[L_{1}\left(t_{2} t\right) Y(t)+Y(t) L_{2}\left(t_{2} t\right)\right]-\left[L_{1}\left(t_{1} t_{0}\right) X\left(t_{2}\right)+X\left(t_{2}\right) L_{2}\left(t_{1} t_{0}\right)\right]\right]$

$$
\begin{aligned}
& -\int_{\mathbb{W}_{\mathrm{W}}}^{\mathrm{T}}\left[\mathbb{K}_{1}\left(t_{s} s\right) \mathbb{Y}(s)+\mathbb{Y}(s) \mathbb{K}_{2}(t, s)\right] \Delta s
\end{aligned}
$$

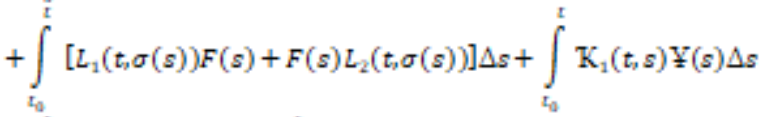

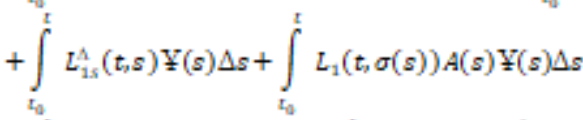

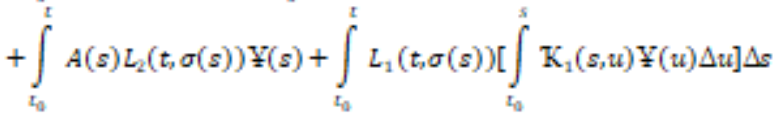




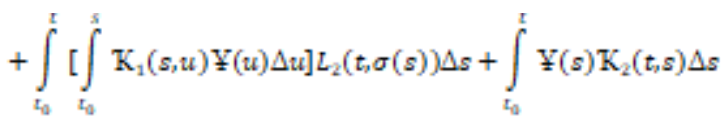

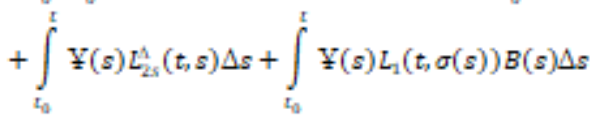

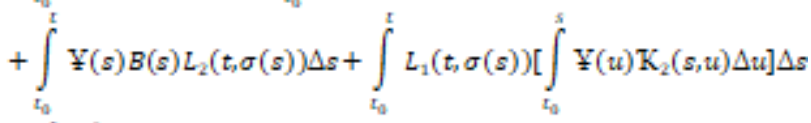

$$
\begin{aligned}
& +\int_{\omega_{1}}^{\mathbb{T}}\left[\int_{L_{1}}^{\infty} \mathrm{Y}(u) \mathbb{K}_{2}(s, u) \Delta u\right] L_{-2}(t, \sigma(s)) \Delta s
\end{aligned}
$$

Set $Q(s)=L_{1}(t, s) Y(s)+Y(s) L_{2}\left(t_{t} s\right)$

$$
\begin{aligned}
Q^{\Delta}(s)= & L_{1 s}^{\Delta}(t, s) \mathbb{Y}(s)+L_{1}(t, \sigma(s)) \Psi^{\Delta}(s)+Y(s) L_{2 s}^{\Delta}(t, s) \\
& +\mathbb{Y}^{\Delta}(s) L_{2}(t, \sigma(s))
\end{aligned}
$$

Integrating between $t_{0}$ to $t$, we have

$$
\begin{aligned}
& Q(t)-Q\left(t_{0}\right)=\int_{t_{0}}^{\pi}\left[L_{1 s}^{\Delta}\left(t_{s} s\right) \mathrm{Y}(s)+L_{1}(t, \sigma(s)) \mathrm{Y}^{\Delta}(s)\right. \\
& \left.+\mathrm{Y}(s) L_{2 s}^{\Delta}(t, s)+\mathrm{Y}^{\Delta}(s) L_{2}(t, \sigma(s))\right] \Delta s \\
& {\left[L_{1}(t, t) \mathrm{Y}(t)+\mathrm{Y}(t) L_{2}(t, t)\right]-\left[L_{1}\left(t, t_{0}\right) \mathrm{X}_{0}+\mathrm{X}_{0} L_{2}\left(t, t_{0}\right)\right]} \\
& =\int_{t_{0}}^{t}\left[L_{1 s}^{\Delta}(t, s) \Psi(s)+L_{1}(t, \sigma(s)) \Psi^{\Delta}(s)\right. \\
& \left.+\mathrm{Y}(s) L_{2 s}^{\Delta}(t, s)+\mathrm{Y}^{\Delta}(s) L_{2}(t, \sigma(s))\right] \Delta s
\end{aligned}
$$

Substituting (13) in (12), we have

$$
\begin{aligned}
& Z(t)=-\int_{t_{0}}^{t} L_{1}\left(t_{v} \sigma(s)\right) X \Psi^{\Delta}(s)-F(s)-A(s) \Psi(s) \\
& \left.-\mathrm{Y}(s) B(s)-\int_{t_{0}}^{s}\left[\mathrm{~K}_{1}(s, u) \mathrm{Y}(u)+\mathrm{Y}(u) \mathrm{K}_{2}(s, u)\right] \Delta u\right\} \Delta s \\
& -\int_{t_{0}}^{t}\left\{Y^{\Delta}(s)-F(s)-A(s) Y(s)-Y(s) B(s)\right. \\
& \left.-\int_{t_{0}}^{g}\left[\mathrm{~K}_{1}(s, u) \mathrm{Y}(u)+\mathrm{Y}(u) \mathbb{K}_{2}(s, u)\right] \Delta u\right\} L_{2}(t, \sigma(s)) \Delta s
\end{aligned}
$$

From (11), we have

$$
\begin{aligned}
Z(t) & =-\int_{t_{0}}^{t} L_{1}(t, \sigma(s)) Z(s) \Delta s-\int_{t_{0}}^{t} Z(s) L_{2}(t, \sigma(s)) \Delta s \\
& =-\int_{t_{0}}^{t}\left[L_{1}(t, \sigma(s)) Z(s)+Z(s) L_{2}(t, \sigma(s))\right] \Delta s
\end{aligned}
$$

Since the solution of the matrix Volterra integral equations are unique, then $Z(t) \equiv 0$ when $Z(t)$ commute with $L_{1}(t, \sigma(s))$ and $L_{2}(t, \sigma(s))$. Therefore

$$
\begin{aligned}
\mathrm{Y}^{\mathrm{a}}(\mathrm{t})= & A(t) \mathrm{Y}(\mathrm{t})+\mathrm{Y}(\mathrm{t}) B(\mathrm{t}) \\
& +\int_{\mathrm{t}_{0}}^{\mathrm{t}}\left[\mathrm{K}_{1}(\mathrm{t}, s) \mathrm{Y}(\mathrm{s})+\mathrm{Y}(\mathrm{s}) \mathrm{K}_{2}(\mathrm{t}, s)\right] \Delta s+F(t)
\end{aligned}
$$

Hence $Y(t)$ is a solution of (1).

Theorem 3.3: Let $C$ and $D$ be two $(n \times n)$ continuous matrix functions and $M, N_{x} \alpha$ and $\beta$ are positive real constants. Assume that the matrix $C(t)$ commutes with $\varepsilon_{C}\left(t_{s} s\right)$ and $\|C(t)\| \leq 1$. If
$\left\|e_{D}(t, s)\right\| \leq N e_{\beta}(t, s)$,

for all $t, s \in \Omega$. Then every solution $\mathrm{X}(t)$ of $(1)$ satisfies

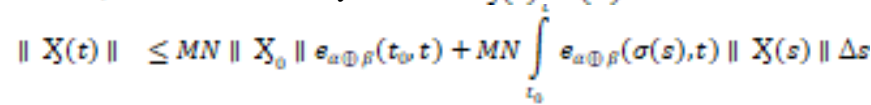

$$
\begin{aligned}
& +M N \int_{0}^{\mathbb{F}} \theta_{M \mathbb{D}}(\sigma(s), t)\|H(s)\| \Delta s
\end{aligned}
$$

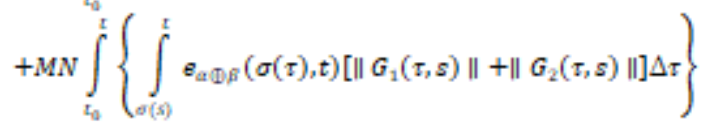

$$
\begin{aligned}
& \mathrm{x}\|\mathrm{X}(s)\| \Delta s
\end{aligned}
$$

Proof:

Let $\mathrm{X}(t)$ be the solution of $(8)$ and $P(t)$ is defined to be $P(t)=e_{C}\left(t_{0}, t\right) X(t) e_{D}\left(t_{0}, t\right)$.

Then

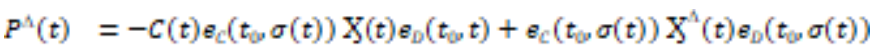
$-e_{i}(t, \sigma(t)) X(t) D(t) e_{D}(t, \sigma(t))$.

Substituting for $\mathrm{X}^{\mathrm{a}}(\mathrm{t})$ from (8), we get

$$
\begin{aligned}
& P^{\prime}(\mathrm{t})=-C(\mathrm{t}) e_{L}\left(\mathrm{t}_{0} \sigma(\mathrm{t})\right) \mathrm{X}(\mathrm{t}) e_{\mathrm{D}}\left(\mathrm{t}_{0}, \mathrm{t}\right) \\
& +e_{-}(t, \sigma(t))\{C(t) X(t)+X(t) D(t) \\
& \left.+\int_{\omega_{1}}^{\pi}\left[G_{1}\left(t_{2} s\right) \mathrm{X}(s)+\mathrm{X}(s) G_{2}\left(\mathrm{t}_{2} s\right)\right] \Delta s+H(\mathrm{t})\right] e_{\mathrm{D}}\left(\mathrm{t}_{\mathrm{w}} \sigma(\mathrm{t})\right) \\
& -\varepsilon_{L}\left(t_{j} \sigma(t)\right) \mathrm{X}(\mathrm{t}) D(\mathrm{t}) e_{\mathrm{D}}(\mathrm{t}, \sigma(\mathrm{t})) \\
& =c(t) e_{L}\left(t_{0} \sigma(t)\right) \mathrm{X}(\mathrm{t})\left[e_{\mathrm{D}}\left(\mathrm{t}_{\mathrm{w}} \sigma(\mathrm{t})\right)-e_{\mathrm{D}}\left(\mathrm{t}_{\mathrm{B}}, \mathrm{t}\right)\right] \\
& +e_{L}(t, \sigma(t)) H(t) e_{D}(t, \sigma(t))
\end{aligned}
$$

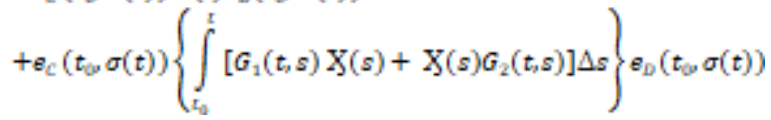

Integration on both sides from $t_{0}$ to $t$, we get

$$
\begin{aligned}
& P(t)-P\left(t_{0}\right)=\int_{t_{0}}^{\pi} C(s) e_{C}\left(t_{0}, \sigma(s)\right) \mathrm{X}(s)\left[e_{D}\left(t_{0}, \sigma(s)\right)-e_{D}\left(t_{0}, s\right)\right] \Delta s \\
& +\int_{t_{0}}^{t} e_{C}\left(t_{0}, \sigma(s)\right) H(s) e_{D}\left(t_{0}, \sigma(s)\right) \Delta s \\
& +\int_{t_{0}}^{t} \theta_{C}\left(t_{0}, \sigma(s)\right)\left\{\int_{t_{0}}^{s}\left[G_{1}(s, \tau) \mathrm{X}(\tau)+\mathrm{X}(\tau) G_{2}(s, \tau)\right] \Delta \tau\right\} \\
& X e_{D}\left(t_{0}, \sigma(s) \Delta s\right. \\
& e_{C}\left(t_{0}, t\right) X(t) e_{D}\left(t_{0}, t\right)-X\left(t_{0}\right) \\
& =\int_{t_{0}}^{t} C(s) e_{C}\left(t_{0}, \sigma(s)\right) \mathrm{X}(s)\left[e_{D}\left(t_{0}, \sigma(s)\right)-e_{D}\left(t_{0}, s\right)\right] \Delta s \\
& +\int_{t_{0}}^{t} e_{C}\left(t_{0}, \sigma(s)\right) H(s) e_{D}\left(t_{0}, \sigma(s)\right) \Delta s \\
& +\int_{t_{0}}^{t} e_{C}\left(t_{0}, \sigma(s)\right)\left\{\int_{t_{0}}^{s}\left[G_{1}(s, \tau) \mathrm{X}(\tau)+\mathrm{X}(\tau) G_{2}(s, \tau)\right] \Delta \tau\right\} \\
& X e_{D}\left(t_{0}, \sigma(s) \Delta s\right.
\end{aligned}
$$

$$
\left\|e_{\mathcal{C}}(t, s)\right\| \leq M e_{\alpha}(t, s) \text {, }
$$




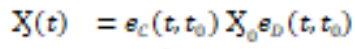

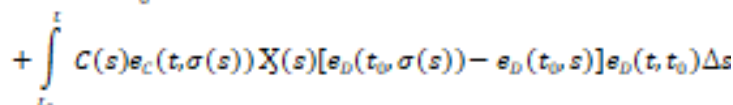

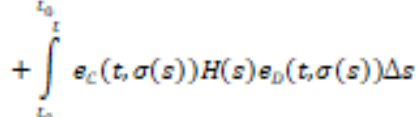

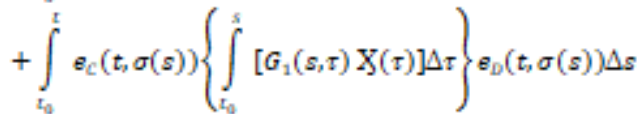

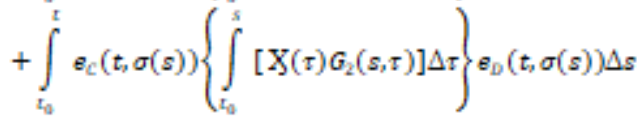

Taking norm on both sides, we get

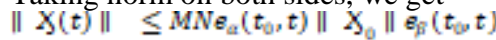

$$
\begin{aligned}
& +M N \int_{\omega_{1}}^{\mathrm{T}} \theta_{u}\left(\sigma(s)_{2} t\right)\|\mathrm{X}(s)\| e_{g}\left(\sigma(s)_{a} t\right) \Delta s
\end{aligned}
$$

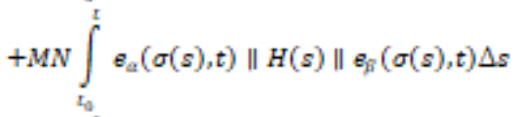

$$
\begin{aligned}
& +M N \int_{0}^{\pi} \theta_{u}(\sigma(s), t)
\end{aligned}
$$

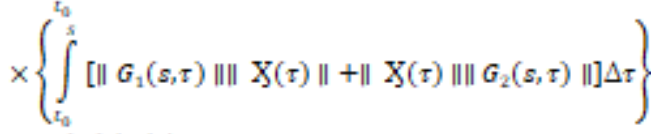

$$
\begin{aligned}
& x e_{p}(\sigma(s), t) \Delta s
\end{aligned}
$$

Using Fubini's Theorem, we obtain

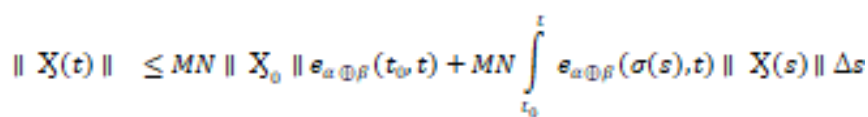

$$
\begin{aligned}
& +M N \int_{\omega_{10}}^{\mathbb{E}} \theta_{w D \beta}(\sigma(s), t)\|H(s)\| \Delta s
\end{aligned}
$$

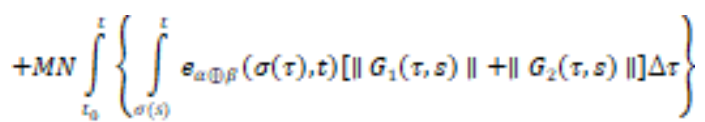

Theorem 3.4: If $L_{1}(t, s)$ and $L_{2}(t, s)$ are continuously differentiable $(n \times n)$ matrix functions on $\Omega$ and also satisfies

(a) the assumptions in Theorem 3.3,

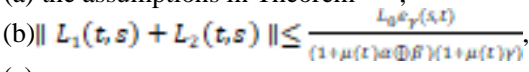

(c)

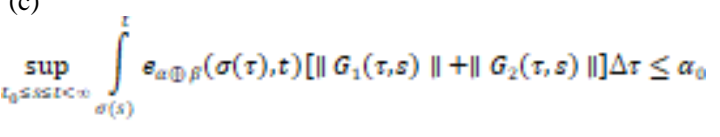

(d) $F(t) \equiv 0$,

where $L_{0}, \gamma(>\alpha \oplus \beta)$ and $\alpha_{0}$ are positive real constants.

If $(\alpha \oplus \beta) \ominus M N\left(1+\alpha_{0}\right)>0$, then every solution $\mathrm{X}(t)$ of $(1)$ tends to zero as $t \rightarrow+\infty$.

Proof : From Theorem 3.2, it is sufficient to prove that as $t \rightarrow+\infty$ any solution of (8) tends to zero exponentially.

From Theorem 3.3 and condition (a), we get

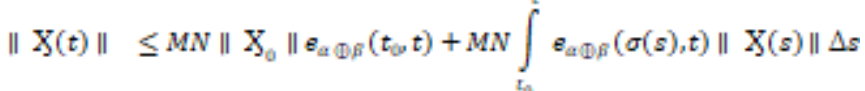
$+M N \int_{0}^{\mathrm{r}} \theta_{m p g}(\sigma(s), t)\|H(s)\| \| \Delta$

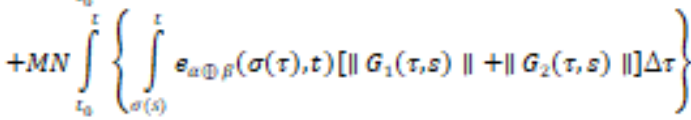
$\mathrm{x}\|\mathrm{X}(s)\| \| \Delta s$

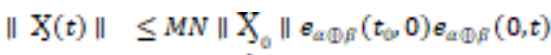

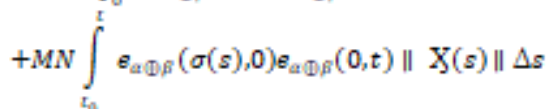
$+M N \int_{0}^{\pi} \theta_{m g}(\sigma(s), 0) e_{m D g}(0, t)\|H(s)\| \Delta s$

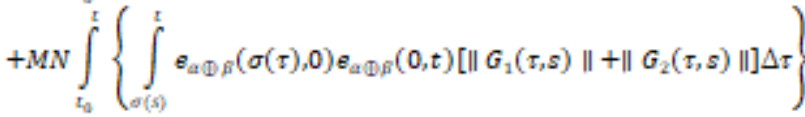
$\mathrm{X}\|\mathrm{X}(s)\| \| s$

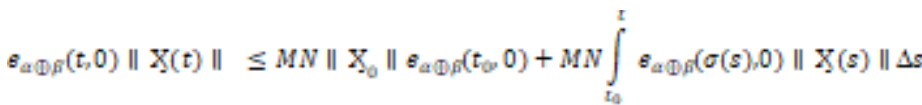
$+M N \int_{\omega_{m}}^{\Gamma} \theta_{u D g}(\sigma(s), 0)\|H(s)\| \Delta s$

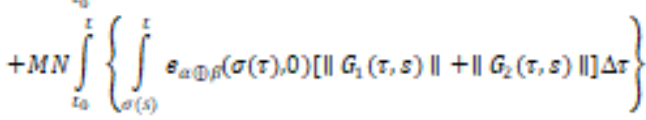
$\mathrm{X}\|\mathrm{X}(\mathrm{s})\| \Delta \mathrm{\Delta}$

Since

$$
\begin{aligned}
& \int_{t_{0}}^{\pi} \theta_{\alpha \oplus \beta}(\sigma(s), 0)\|H(s)\| \Delta s \\
& =\int_{\sigma_{0}}^{t} e_{\alpha \oplus \beta}(\sigma(s), 0)\left[\left\|L_{1}\left(s, t_{0}\right)\right\|\left\|\mathrm{X}_{0}\right\|+\left\|L_{2}\left(s, t_{0}\right)\right\|\left\|\mathrm{X}_{0}\right\|\right] \Delta s \\
& =\left\|\mathrm{X}_{0}\right\| \int_{\mathrm{t}_{0}}^{\mathrm{t}} e_{\alpha \oplus \beta}(\sigma(s), 0)\left[\left\|L_{1}\left(s_{s}, t_{0}\right)\right\|+\left\|L_{2}\left(s_{s}, t_{0}\right)\right\|\right] \Delta s \\
& \leq\left\|\mathrm{X}_{0}\right\| \int_{\mathrm{t}_{0}}^{\mathrm{t}} \frac{e_{\alpha \oplus \beta}(\sigma(s), 0) L_{0} \epsilon_{\gamma}\left(t_{0}, s\right)}{(1+\mu(s) \alpha \oplus \beta)(1+\mu(s) \gamma)} \Delta s \\
& =\left\|\mathrm{X}_{0}\right\| L_{0} \int_{t_{0}}^{t} \frac{e_{\alpha \oplus \beta}(\sigma(s), 0) \theta_{\gamma}\left(t_{0}, 0\right) e_{\gamma}(0, s)}{(1+\mu(s) \alpha \oplus \beta)(1+\mu(s) \gamma)} \Delta s \\
& =\left\|\mathrm{X}_{0}\right\| L_{0} e_{\gamma}\left(t_{0}, 0\right) \int_{t_{0}}^{t} \frac{e_{\alpha \oplus \beta}(\sigma(s), 0) e_{\gamma}(0, s)}{(1+\mu(s) \alpha \oplus \beta)(1+\mu(s) \gamma)} \Delta s \\
& =\left\|\mathrm{X}_{0}\right\| L_{0} e_{\gamma}\left(t_{0}, 0\right) \int_{t_{0}}^{t} \frac{(1+\mu(s) \alpha \oplus \beta) e_{\alpha \oplus \beta}(s, 0) e_{\gamma}(0, s)}{(1+\mu(s) \alpha \oplus \beta)(1+\mu(s) \gamma)} \\
& =\left\|\mathrm{X}_{0}\right\| L_{0} e_{Y}\left(t_{0}, 0\right) \int_{t_{0}}^{t} \frac{e_{\alpha \oplus \beta}(s, 0)}{(1+\mu(s) \gamma) e_{Y}\left(s_{0}, 0\right)} \Delta s \\
& =\left\|\mathrm{X}_{0}\right\| L_{0} \epsilon_{Y}\left(t_{0}, 0\right) \int_{t_{0}}^{t} \frac{\theta_{\alpha \oplus \beta}(s, 0)}{\theta_{\gamma}(\sigma(s), 0)} \Delta s
\end{aligned}
$$




$$
=\left\|\mathrm{X}_{0}\right\| L_{0} \theta_{Y}\left(t_{0}, 0\right) \int_{t_{0}}^{\pi} \frac{((\alpha \oplus \beta)-\gamma) e_{\alpha \oplus \beta}(s, 0)}{((\alpha \oplus \beta)-\gamma) e_{\gamma}(\sigma(s), 0)} \Delta s \text { References }
$$

From lemma 2.8, we obtain

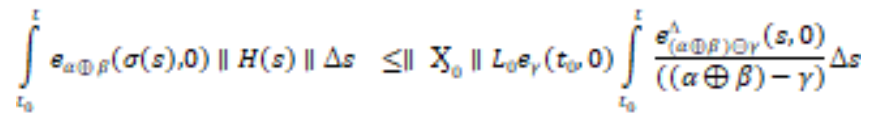

Since $\gamma>(\alpha \oplus \beta)$, we have

$$
\int_{t_{0}}^{t} e_{\alpha \oplus \beta}(\sigma(s), 0)\|H(s)\| \Delta s \quad \leq\left\|\mathrm{X}_{0}\right\| L_{0} \frac{e_{(\alpha D}\left(\gamma-\mathrm{t}_{0} 0\right)}{\|(\alpha \oplus \beta))}
$$

From (14), (b), (c) and (d), we have

$\theta_{\alpha \oplus \beta}(t, 0)\|X(t)\| \leq M N\left\|X_{0}\right\| \epsilon_{\alpha \oplus \beta}\left(t_{0}, 0\right)$

$$
\begin{aligned}
& +M N\left\|X_{0}\right\| L_{0} \frac{e_{[\alpha \oplus \beta)}\left(t_{0}, 0\right)}{(\gamma-(\alpha \oplus \beta))} \\
& +M N \int_{t_{0}}^{t} e_{\alpha \oplus \beta}(s, 0)\|X(s)\| \Delta s \\
& +M N \int_{t_{0}}^{t} \alpha_{0} \theta_{\alpha \oplus \beta}\left(s_{x} 0\right)\|X(s)\| \Delta s
\end{aligned}
$$

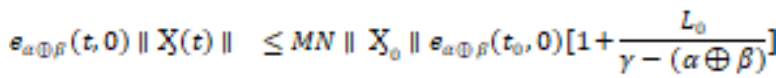

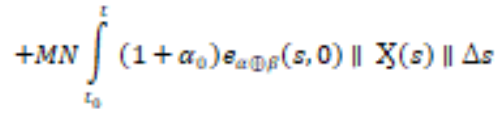

By Lemma 2.10, we have

$$
\begin{aligned}
& \theta_{\alpha \oplus \beta}(t, 0)\|\mathrm{X}(t)\| \leq M N\left\|\mathrm{X}_{0}\right\|\left[1+\frac{L_{0}}{\gamma-(\alpha \oplus \beta)}\right] \\
& x e_{\alpha \oplus \beta}\left(t_{0}, 0\right) e_{M N 1+c_{\alpha}}\left(t, t_{0}\right) \\
& \text { II } X(t) \|] \leq M N\left\|X_{0}\right\|\left[1+\frac{L_{0}}{\gamma-(\alpha \oplus \beta)}\right]
\end{aligned}
$$

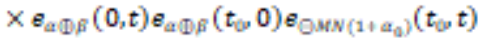

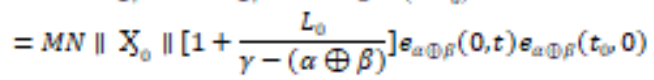

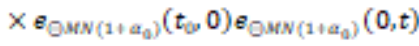

$$
\begin{aligned}
& =M N\left\|\mathrm{X}_{\omega}\right\|\left[\mathrm{I}\left[1+\frac{L_{0}}{\gamma-(\alpha \oplus \beta)}\right]\right.
\end{aligned}
$$

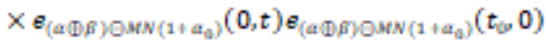

By Lemma 2.9, we have

So we obtain

$$
\theta_{(\alpha \oplus \beta) \in M N\left(1+\alpha_{0}\right)}(0, t) \leq \frac{1}{\left((\alpha \oplus \beta) \ominus M N\left(1+\alpha_{0}\right)\right) t}{ }^{x}
$$

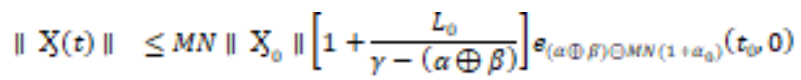

\section{$x \frac{1}{\left((\alpha \oplus \beta) \Theta M N\left(1+\alpha_{2}\right)\right) t}$}

Since $(\alpha \oplus \beta) \ominus M N\left(1+\alpha_{0}\right)>0$.

Hence we get the required result.

\section{Conclusion}

The present paper is focussed on Lyapunov type matrix Volterra integro-dynamic system on time scales. An equivalence relation between Volterra integro-differential system and differential system is established. The conditions for the asymptotic stability of the integro-differential system on time scales are obtained.
[1] M. Bohner, A. Peterson, Dynamic Equations on Time Scales, Birkhauser, Boston, (2001).

[2] T.A. Burton, "Stability theory for Volterra equations", J. Diff Equs, Vol.32, (1979), pp.101-118.

[3] T.A. Burton, "Integro differential equations", Proc. Amer. Math. Soc Vol.79, (1980), pp.393-399.

[4] S.I. Grossman, R. K. Miller, "Perturbation theory for Volterra integro-differential system”, J. diff. Equs Vol.8, (1970), pp.451-474.

[5] S. Hilger, Ein Mabkettenkalkul mit Anwendung auf Zentrumsmannigfaltigkeiten, $\mathrm{PhD}$ thesis, Universitat Wurzburg, (1988).

[6] B. Karpuz, Basics of volterra integral equations on time scales, (2011).

[7] T. Kulik, C.C. Tisdell, "Volterra integral equations on time scales", Int. J. Difference Equ, Vol.3, (2008) pp.103-133.

[8] V. Lupulescu, K. Ntouyas, and A.Younus, "Qualitative aspects of a Volterra integro-dynamic system on time scales", Electronic Journal of Qualitative Theory of Differential Equations, Vol.5, (2013), pp.1-35.

[9] V. Volterra, Theory of functionals and integral and integro-differential equations, Dover, New York, (1950). 